\title{
Impact of Congestion Management Mechanism on Contract Revenue of Generator
}

\author{
Liu Jun \\ State Grid Zhejiang Economic \\ Research Institute \\ Hangzhou, China. \\ email:liuliu2606@sina.com
}

\author{
Ding Jie \\ Nanjing Normal University \\ Nanjing, China. \\ 540140028@qq.com
}

\author{
Huang Weiqun \\ State Grid Ningbo Power Supply \\ Company \\ Ningbo, China \\ hwq6921@163.com
}

\author{
Zhou Yuan \\ Zhejing Gongshang University \\ Hangzhou, China \\ abalezf@foxmail.com
}

\author{
Sun Renjie \\ Nanjing Normal University \\ Nanjing, China. \\ 645840917@qq.com
}

\begin{abstract}
At present, with deepening reform of electricity market in China, the transmission network's operating environment is complex, and the issue of transmission congestion has received increasing attention. The typical congestion management methods are classified to congestion pricing method and congestion correction method. Besides, congestion management methods of abroad typical electricity markets are summarized. The impact of congestion management mechanism on the contract revenue of power generators are analyzed according to the example of the UK and Australia power market. The relevant case studies verify and conclude that the impact of congestion management on contract revenue is mainly based on whether the compensation for the constrained-on units. At the same time, some suggestions on congestion management mechanism of power market in China are put forward.
\end{abstract}

Keywords-congestion management, nodal price, rescheduling, contract revenue, constrained-on units, contract for difference

\section{INTRODUCTION}

With deepening reform of electricity market in China, market entities and market trading varieties are gradually diversified, and the proportion of energy trading in cross-regional and inter-provincial transactions is increasing. The operating environment of transmission networks is becoming more and more complex, and the issue of transmission congestions has received increasing attention [1-4].

When the power load flow determined by the electricity market transaction's FPN exceeds the power transmission capacity, resulting in one or more grid security constraints, the transmission congestions will appear [5-7]. The main measures are to increase the source at some nodes and reduce the source at other nodes. The targets of congestion management are (1) developing active power generation plans that meets the system safety standards; (2) providing reasonable economic signals that are consistent with active power generation plans; and (3) reducing risk of congestion with effective economic methods.[2]. At present, many scholars have conducted a lot of research on congestion management and its pricing mechanism. Literature [3] summarizes the optimal congestion management methods under different transaction modes, expounds the corresponding congestion adjustment objectives, and summarizes dynamic congestion, cross-regional transaction congestion management, congestion pricing and congestion cost allocation mechanism. The literature [8-9] divides the congestion management into two categories from the technical and non-technical perspectives, and divides the non-technical perspective into two parts based on the electricity market and the non-electricity market, and summarizes different congestion management methods in the past decades in detail. Besides, the paper introduces relevant national congestion management measures. However, most of the current researches focus on the impact of congestion management on the operation of the spot market, and rarely involve its impact on power contracts.

Based on the classification of the congestion management mechanism, this article summarizes the current congestion management measures in some typical foreign electricity markets, and analyzes the impact of congestion management based on the contractor's return on the electricity market in the UK and Australia. The paper also puts forward some suggestions on the congestion management mechanism of China's electricity market.

\section{ClASSIFICATION OF CONGESTION MANAGEMENT MECHANISMS}

Congestion management requires different market mechanisms in different electricity market modes of different countries, namely how to guide market members to adjust their power generation source and power load. This article divides the congestion management market mechanism into two main categories: One is the congestion correction method, also known as the rescheduling method. The method is divided into two phases. The first phase firstly clears the market without grid security constraints. Then, the dispatching institute judges whether the load flow corresponding to the market clearing result causes blocking. If there is a transmission congestion, then in the next stage it will adjust the power generation plan (or electricity consumption plan) formed by the generator (or user) in the first unconstrained clearing, that is, adjust the power source 
and sink at a specific location, and give the generator (or user) a certain cost. Congestion management in the UK electricity market is a typical example of this approach. Regional electricity prices combined with counter-trading transactions in the Nordic electricity market are also covered by such methods.

The other is the congestion pricing method, which can also be called the locational marginal pricing (LMP) mechanism, which considers the cyber security constraints at the market clearing, and restricts the electricity transactions of market members, and only the power transactions that can produce feasible load flow can be allowed. Once the transmission congestion appears, the market clearing prices of the different nodes are different to reflect the different costs that meet the new load requirements at different nodes. Node electricity prices are used in most of the US electricity market, such as the US PJM, New York and other electricity markets. The regional electricity price mechanism adopted in the Australian national electricity market is a simplified version of the mechanism and will gradually transition to node electricity prices in the future.

\section{CONGESTION MANAGEMENT IN TyPiCAL ForEIGN ELECTRICITY MARKETS}

\section{A. Congestion Management in the Australian National Electricity Market}

The National Electricity Market (NEM) adopts the power pool model. The market operation center determines the generator (station) output based on the node price principle, quotation of the power generation company, the power load prediction and the grid operation status (automatically done by the NEMDE scheduling tool). However, the electricity price signal does not adopt the node electricity price method which can directly reflect the congestion cost, but is divided into five electricity price zones according to the administrative state. The marginal price of the electricity supply in the price zone is the price of the price zone market, that is, for each additional megawatt of load in the Price area, the additional generating capacity will be quoted, and the clearing price will be defined on the Regional Reference Node-RRN, and is called Regional Reference Price-RRP. Market members in each region are settled according to RRP of their region.

\section{B. UK electricity market congestion management}

In the UK electricity market (England and Wales), the power pool model was put into use in 1989-2000. The power pool center will firstly make unconstrained clearing based on the generator's bid, and then on the basis of System Marginal Price(SMP), transmission congestion is treated by real-time constrained clearing (rescheduling). Since 2000, the UK electricity market (England and Wales) has abandoned the power pool model and adopted the NETA (New Electricity Trading Arrangements) model. In 2005, the Scottish Power Grid joined, that is, the NETA model was implemented throughout the UK, called the BETTA (British Electricity Trading and Transmission Arrangements) model. The NETA model mainly uses bilateral contract transactions as the basis. The generators will schedule their own units, and the dispatching institute NGET will no longer centrally dispatch them. NGET only plays the role of balancing the market. In this mode, the scheduling NGET solves the unbalanced power and operating constraints of the system by receiving the Offer and Bid quotations and rescheduling in the balanced market. In the NETA mode, the "two-time bids, two-time clearings" are used, and a bid is made before the unconstrained clearing. In the balanced market, the market members are required to declare the Offer and Bid quotes to deal with the congestion and power imbalance problems.

\section{ANALYSIS OF THE IMPACT OF CONGESTION MANAGEMENT ON GENERATORS' CONTRACT REVENUE}

Congestion management measures may have impacts on contract revenue for power producers that reduce power generation when they are unconstrained due to congestion. This article mainly selects the electricity market in the UK and Australia for analysis. The Nordic market is similar to the UK NETA model and is no longer analyzed.

\section{A. The impact of the UK electricity market congestion} management model on the power producers's revenue

The contract market in NETA mode mainly includes medium and long-term contract transactions and short-term spot contract transactions. Medium and long-term contracts are mainly completed through bilateral over-the-counter (OTC) transactions. Short-term spot transactions are mainly done by the power trading center PX. Both of them do not count the security constraints of the network at the time of the transaction.

One hour before the trading interval, the contract market closed into a balancing mechanism. Both parties are required to submit the contracted energy and FPN for the trading session before the gate closure, and may submit the Offer and Bid declarations for the increase or decrease. In the balancing mechanism, the scheduling institute NGET solves the unbalanced energy and operational constraints of the system by receiving the offer and Bid of the market members. Among them, Bid is the quotation that the market members pay the dispatcher to carry out the unit's output or the demand increase load; Offer is the quotation that the market members charge the dispatch, and the unit's output is increased or the demand is reduced. Units that reduce power generation based on Bid may be referred to as constrained-on units, and units that increase power generation according to Offer may be referred to as constrained-off units, and their respective contract energy settlements are not affected by the adjustment.

Since the Bid price of the units set is generally lower than the marginal cost of power generation, constrained-off units are equivalent to purchasing the power in the balancing market to complete its own contracted energy in a way that is lower than the marginal cost of power generation, so the contract revenue will not be reduced due to congestion management.

\section{B. Impact of Australian NEM congestion management on generators' revenue on CFDs}

It is stated in Section 3 that the Australian NEM determines the unit output based on the node price principle, but uses the price of the reference node in the price area as the settlement price RRP of the unit in the area. Therefore, when there is a congestion in the price zone, the relevant affected units are limited to the constrained-on and the constrained-off.

Constrained-on: The bid is higher than the RRP in the 
area, but it is dispatched.

Constrained-off: The bid is lower than the RRP in the area, but it cannot be dispatched.

For the constrained-off units, the expected revenue under the RRP price is reduced due to the congestion, but the current market rules of Australia NEM have no compensation for the constrained-on and constrained-off units. If the constrained-off unit has signed a CFD, the settlement of the financial contract needs to refer to the spot market price, especially at the higher RRP price, which will cause financial risks to the constrained-off unit in the contract market.

\section{EXAMPLE ANALYSIS}

\section{A. UK Electricity Market Example}

The impact of UK power market congestion management on the constrained-off unit contracts is analyzed using the simple two-node example in Figure 1. The capacity of the two units at node $A$ is $P_{\text {A1max }}=290 \mathrm{MW}$ and $\mathrm{P}_{\mathrm{A2max}}=30 \mathrm{MW}$ respectively. The capacity of one unit at node $B$ is $P_{B \max }=120 \mathrm{MW}$, and the power generation cost of the corresponding generator is $\mathrm{P}_{\mathrm{A} 1}=150 \mathrm{E} / \mathrm{MWh}$, $\mathrm{P}_{\mathrm{A} 2}=220 £ / \mathrm{MWh}$ and $\mathrm{P}_{\mathrm{B}}=300 £ / \mathrm{MWh}$, the line $\mathrm{AB}$ transmission limit is $70 \mathrm{MW}$, and the loads of the two nodes $\mathrm{A}$ and $\mathrm{B}$ are $\mathrm{P}_{\mathrm{LA}}=180 \mathrm{MW}$ and $\mathrm{P}_{\mathrm{LB}}=120 \mathrm{MW}$ respectively.

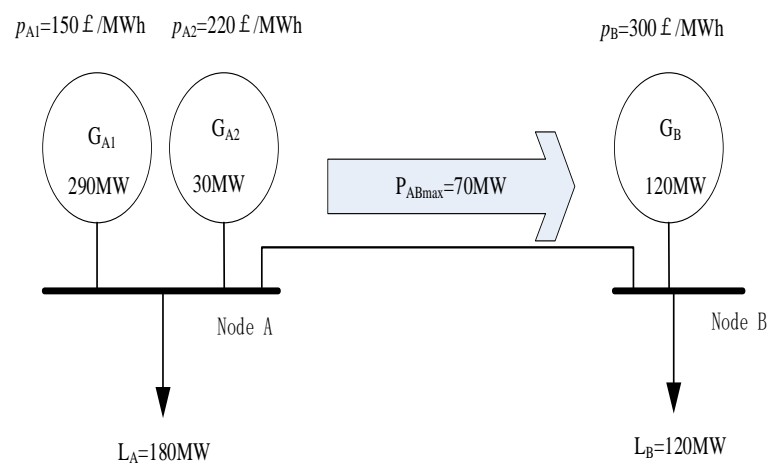

Figure.1. Uk Power Market Congestion Management Two-Node Example Diagram

Assume that the power producer $\mathrm{G}_{\mathrm{A} 1}$ has a contract volume and FPN of $280 \mathrm{MW}$ during the trading interval, the contract price $\mathrm{p}_{\mathrm{C}}=200 \mathrm{f} / \mathrm{MWh}$, and the bidding price in the balance mechanism is $\mathrm{p}_{\mathrm{b}}=145 \mathrm{f} / \mathrm{MWh}$.

Without considering the power deviation,

The revenue of the generator $G_{A 1}$ when there is no congestion is

$$
280 \times(200-150)=14000(£)
$$

If there is a congestion, the generator will reduce $30 \mathrm{MW}$ according to the schedule, then the revenue of the generator $\mathrm{G}_{\mathrm{A} 1}$ is

$280 \times 200-250 \times 150-30 \times 145=14150(£)$

It can be seen that since the bidding price is generally lower than the power generation cost of the power producer, the reduced power generation of the constrained-off unit does not reduce its contract revenue, and may even obtain higher revenue.

\section{B. Australian Electricity Market Example}

In Figure 2, both nodes A and B are in the same price zone of the Australian electricity market. The dispatching institute will determine the output according to the node price principle. The market only needs to clear once, that is, it has the constrained clearing.

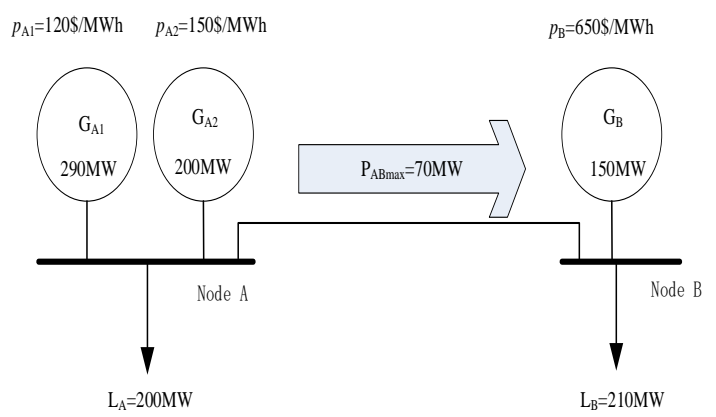

Figure.2. Australian Electricity Market Congestion Management Two-Node Example Diagram

The unit is quoted according to the marginal cost, $p_{\mathrm{A} 1}=120 \$ / \mathrm{MWh} 、 p_{\mathrm{A} 2}=150 \$ / \mathrm{MWh}$ and $p_{\mathrm{B}}=650 \$ / \mathrm{MWh}$. If point $B$ is the reference point of the price zone, the electricity price $p_{\mathrm{RRP}}$ of this area is 650\$/MWh. $p_{\mathrm{A} 2}<p_{\mathrm{RRP}}$, but the unit $G_{\mathrm{A} 2}$ cannot be dispatched because of the congestion, it becomes constrained-off unit. If it signs a two-way CFD, the settlement reference price is $p_{\mathrm{RRP}}$, the contract price is $p_{C}=200 \$ / M W h$, and the contract power is $100 \mathrm{MW}$, then the CFD income of $G_{\mathrm{A} 2}$ is

$$
100 \times(200-650)=-45000(\$)
$$

And its revenue in the spot market is $0 \$$, so the generator unit $\mathrm{G}_{\mathrm{A} 2}$ caused a large economic loss due to the signing of the CFD.

In practice, generators consider this risk to tend to reduce the amount of CFDs or sign higher prices when signing CFDs. In addition, in order to avoid becoming a constrained-on or constrained-off unit in the spot market, the unit may adopt some extreme strategic bidding behaviors, quoted at the lowest price $(-\$ 1000 / \mathrm{MWh})$ to avoid becoming a constrained-off unit, at the highest price (\$1000/MWh) quote to avoid becoming a constrained-on unit. This bidding behavior will affect the efficiency of the entire market.

\section{CONCLUSION}

It can be seen from the comparison between the UK and Australia electricity markets that the impact of congestion management on contract revenue is mainly whether to compensate for the constrained-off units. Both methods have their own advantages and disadvantages.

The UK adopts a compensation method, which has the advantage of not affecting the revenue of the unit and facilitating the power producer to accept market dispatch 
results. The disadvantage is the lack of the necessary economic signals to alleviate the congestion.

Australia adopts a non-compensation method, which has the advantage of providing an economic signal to the market, guiding the generators to avoid constructing new units at the nodes where the units are constrained-off, and to build low-cost units at the nodes where the units are constrained-on. But the disadvantage is that the unit is not willing to accept the scheduling results, may take some game behavior in the spot and contract market, affecting market efficiency.

The congestion management mechanism is one of the key issues in the construction of the spot market. If the reasonable contract revenue of the power producer cannot be guaranteed, it will inevitably affect the construction of the medium and long-term market. The author suggests that for the market adopting the rescheduling mechanism, the UK's compensation model can be used for reference; In the unified price settlement market, if the adoption of Australia's uncompensated method may not be conducive to power generation enterprises to accept the power market reform, it may consider adopting the UK's compensation method, based on the difference between the marginal cost and the market electricity price approved by the unit, the relevant costs may be considered through the user's share of recycling, the gradual transition to the node price in the future.

\section{ACKNOWLEDGMENT}

This work was supported in part by State Grid Zhejiang
Electric Power Company Project named research on key issues of the initial power market contract system and market entity ecological circle construction in Zhejiang under Grant Number 5211JY7000W.

\section{REFERENCES}

[1] He Hui. Investigation of transmission congestion and relate problems of electrical system[D]. Zhejiang University,2005.

[2] Sun D. Market-based congestion management[C] Power Engineering Society Winter Meeting. IEEE, 2002:127 vol.1.

[3] Zhang Yongping, Jiao Lianwei, Chen Shousun, et al. A survey of transmission congestion management in electricity markets[J]. Power System Technology, 2003.

[4] Huang hanying. Research on congestion management methods in power market [D]. North China electric power university (Beijing),2017.

[5] Nandini S, Suganya P, Lakshmi M K M. Congestion management in transmission lines considering demand response and facts devices[J]. Power System, 2010.

[6] Chung H M, Su C L, Wen C K. Dispatch of generation and demand side response in regional grids[C] IEEE, International Conference on Environment and Electrical Engineering. IEEE, 2015:482-486.

[7] Nappu M B, Arief A. Network losses-based economic redispatch for optimal energy pricing in a congested power system[J]. Energy Procedia, 2016, 100:311-314.

[8] Abhishek Saxena, Seema N. Pandey, Srivastava Laxmi "Congestion management in open access: a review" Int J Sci, Eng Technol Res (IJSETR), 2 (4) (2013)

[9] Pillay A, Karthikeyan S P, Kothari D P. Congestion management in power systems - A review[J]. International Journal of Electrical Power \& Energy Systems, 2015, 70:83-90. 